\title{
Saccades and handedness interact to affect scene memory
}

\author{
Timothy M Ellmore ${ }^{\text {Corresp., }}{ }^{1}$, Bridget Mackin ${ }^{1}$, Kenneth Ng $^{1}$ \\ ${ }^{1}$ Department of Psychology, City University of New York, City College, New York, New York, United States \\ Corresponding Author: Timothy M Ellmore \\ Email address: tellmore@ccny.cuny.edu
}

Repetitive saccades benefit memory when executed before retrieval, with greatest effects for episodic memory in consistent-handers. Questions remain including how saccades affect scene memory, an important visual component of episodic memory. The present study tested how repetitive saccades affect working and recognition memory for novel scenes. Handedness direction (left-right) and degree (strong/consistent vs. mixed/inconsistent) was measured by raw and absolute laterality quotients respectively from an 8-question handedness inventory completed by 111 adults. Each then performed either 30 seconds of repetitive horizontal saccades or fixation before or after tasks of scene working memory and scene recognition. Regression with criterion variables of overall percent correct accuracy and d-prime sensitivity showed that when saccades were made before working memory, there was better overall accuracy as a function of increased direction but not degree of handedness. Subjects who made saccades before working memory also performed worse during subsequent recognition memory, while subjects who fixated or made saccades after the working memory task performed better. Saccades made before recognition resulted in recognition accuracy that was better (Cohen's $d=0.3729$ ), but not significantly different from fixation before recognition. The results demonstrate saccades and handedness interact to affect scene memory with larger effects on encoding than recognition. Saccades before scene encoding in working memory are detrimental to short- and long-term memory, especially for those who are not consistently right-handed, while saccade execution before scene recognition does not appear to benefit recognition accuracy. The findings are discussed with respect to theories of interhemispheric interaction and control of visuospatial attention. 
1

2

3

4

5

6

7

8

9

10

11

12

13

14 City University of New York, City College

15 New York, New York, United States of America

16

17

18

19

20

${ }^{*}$ Corresponding author

21 Timothy M. Ellmore, Ph.D.

22 Associate Professor

23 Department of Psychology

24 The City College of the City University of New York

25 E-mail: tellmore@ccny.cuny.edu

26 Phone: (212) 650-5714

27 Fax: (212) 650-5659

29 
30

31

32

33

34

35

36

37

38

39

40

41

42

43

44

45

46

47

48

49

50

51

52

53

\begin{abstract}
Repetitive saccades benefit memory when executed before retrieval, with greatest effects for episodic memory in consistent-handers. Questions remain including how saccades affect scene memory, an important visual component of episodic memory. The present study tested how repetitive saccades affect working and recognition memory for novel scenes. Handedness direction (left-right) and degree (strong/consistent vs. mixed/inconsistent) was measured by raw and absolute laterality quotients respectively from an 8-question handedness inventory completed by 111 adults. Each then performed either 30 seconds of repetitive horizontal saccades or fixation before or after tasks of scene working memory and scene recognition. Regression with criterion variables of overall percent correct accuracy and d-prime sensitivity showed that when saccades were made before working memory, there was better overall accuracy as a function of increased direction but not degree of handedness. Subjects who made saccades before working memory also performed worse during subsequent recognition memory, while subjects who fixated or made saccades after the working memory task performed better. Saccades made before recognition resulted in recognition accuracy that was better (Cohen's $d=0.3729$ ), but not significantly different from fixation before recognition. The results demonstrate saccades and handedness interact to affect scene memory with larger effects on encoding than recognition. Saccades before scene encoding in working memory are detrimental to short- and long-term memory, especially for those who are not consistently right-handed, while saccade execution before scene recognition does not appear to benefit recognition accuracy. The findings are discussed with respect to theories of interhemispheric interaction and control of visuospatial attention.
\end{abstract}


54 Introduction

55

56

57

58

59

60

61

62

63

64

65

66

67

\section{Saccade Induced Retrieval Enhancement}

As few as thirty seconds of horizontal bilateral saccadic eye movements before testing selectively enhances explicit memory, most notably episodic memory retrieval for laboratory and everyday events (Christman et al. 2003). This has been termed saccade induced retrieval enhancement (SIRE) by Lyle and colleagues (Lyle et al. 2008a). The benefits of SIRE appear to be specific to retrieval. Early preliminary findings reported that eye movements before encoding hurt subsequent memory performance (Christman \& Butler 2005). Later experiments by others showed that the benefits of horizontal eye movements are seen when they immediately precede episodic memory retrieval but not when they precede encoding (Brunye et al. 2009). One hypothesis is that horizontal eye movements enhance interhemispheric interaction, which is associated with superior episodic memory (Christman \& Propper 2001). Increased interhemispheric interaction has also been related to decreased false memories in a semantic associates paradigm (Christman et al. 2004).

Bilateral eye movements also appear to enhance some types of recognition memory. Subjects who made bilateral eye movements were more likely to correctly recognize previously presented words, but less likely to falsely recognize critical non-studied associates (Parker \& Dagnall 2007). Parker et al conducted multiple experiments in a study investigating effects of bilateral saccadic eye movements on item, associative, and contextual information. In tests of item recognition they found bilateral horizontal eye movements versus no eye movements enhanced item recognition by increasing hit rate and decreasing false alarms; an additional remember-know analysis showed eye movements increased "remember" responses (Parker et al. 2008). For associative recognition, they found bilateral eye movements increased correct responses to intact 
79 pairs and decreased false alarms to rearranged pairs (Parker et al. 2008). Bilateral eye movements

80 also increased correct recall for both intrinsic (color) and extrinsic (spatial location) context

81 (Parker et al. 2008).

82 More recently, the horizontal SIRE effect has been extended beyond the visual domain in 83 a study that showed retrieval enhancement in the somatosensory system after alternating left-right

84 tactile stimulation (Nieuwenhuis et al. 2013). In what appears to be the only study of the effects of 85 horizontal saccadic eye movements on the retrieval of landmark shape and location information, 86 increased recognition sensitivity and decreased response times were reported in a spatial memory 87 test with effects only seen when eye movements preceded episodic memory retrieval, but not when they preceded encoding (Brunye et al. 2009). Furthermore, these same authors found that eye movements were only beneficial in an old-new recognition paradigm, which purportedly involves more elaborate recollective processing and presumably demands a high degree of right and left-

91 hemisphere activity, compared to a forced-choice recognition test, which is thought to be more 92 dependent on familiarity. (Lyle \& Jacobs 2010; Propper \& Christman 2008; Stickgold 2002), there are only a few studies employing neuroscientific methods to measure brain-based correlates of bilateral saccades. Bilateral eye movements have been reported to have significant effects on interhemispheric

97 coherence in the gamma band as measured by EEG (Propper et al. 2007). But a more recent EEG 98 study found scant evidence that eye movements altered interhemispheric coherence or that 99 improvements in recall were correlated with changes in coherence (Samara et al. 2011). 

recall and recognition with strongly right-handed (SR) and non-strongly right handed (nSR)

104 subjects found that eye movements largely benefited the former while it was shown to be 105 somewhat detrimental to the latter (Lyle et al. 2008a). This finding was initially interpreted to 106 support the hemispheric interaction hypothesis. Better memory without eye movements is thought

107 to exist in nSR individuals because nSR handedness is thought to be a behavioral marker for greater 108 interhemispheric interaction. Consistent with this idea, middle-age nSR subjects perform better on 109 tasks like paired associate recall and source memory, which likely depend on hemispheric

110 interaction, compared to tasks like face recognition and forward digit span which depend less on

111 hemispheric interaction (Lyle et al. 2008b). For example one study (Lyle \& Orsborn 2011)

112 required subjects to classify faces as famous or novel with face presentation occurring in the left

113 and right visual fields simultaneously (bilaterally) or in one field only (unilaterally). Famous faces

114 were classified more quickly and accurately during bilateral presentation, reflecting that 115 interhemispheric interaction facilitates famous face recognition, but neither inconsistent

116 handedness nor saccades increased the size of bilateral gain.

117 Strong right-handedness, which is argued to be associated with decreased interhemispheric

118 interaction, was associated with higher rates of false memories while bilateral saccades were

119 associated with fewer false memories. Other evidence suggests that mixed-handers display better

120 episodic memory in comparison with strong right-handers on assessments of explicit word recall

121 and recall of real world events, but when corrected scores are analyzed handedness does not

122 influence implicit word fragment completion (Propper et al. 2005). Mixed-handedness and

123 bilateral saccadic eye movements have also both been associated with an earlier offset of childhood

124 amnesia and support the idea that interhemispheric interaction exerts effects on retrieval but not 
125 encoding of episodic memories. Mixed-handed subjects also demonstrate greater autobiographical

126 recollection on components of seeing hearing and emotion compared to right-handed individuals,

127 and $30 \mathrm{sec}$ of bilateral eye movements induce greater levels of autobiographical recollection across

128 a range of components (Parker \& Dagnall 2010).

130 From Interhemispheric Interaction to Top Down Control

131 A recent experiment in which subjects were shown arrays of lateralized letters and were

132 asked whether target letters matched either of two probe letters, saccades were reported to enhance

133 retrieval by increasing interaction among brain hemispheres (Lyle \& Martin 2010). Matching

134 targets and probes were presented to either the same hemisphere or to separate hemispheres.

135 Interhemispheric interaction was required on the across-hemisphere trials and intrahemispheric

136 processing was required on the same hemisphere trials. Increased match detection accuracy was

137 found on the within-hemisphere trials as a function of pre-task eye-movements suggesting 138 saccades enhance intrahemispheric processing but not interhemispheric interaction. The nSR

139 subjects showed higher across-hemisphere accuracy suggesting that an absence of strong right-

140 handedness may reflect greater interhemispheric interaction. A recent comparison of consistent

141 and inconsistent left- and right-handers on associative memory tests after saccade or no-saccade

142 conditions showed that saccades enhanced retrieval for consistent-handers only, but impaired

143 retrieval for inconsistent-handers (Lyle et al. 2012). This important study established that

144 handedness consistency, regardless of left or right direction, is an important factor to consider 145 when studying memory.

146 Given the lack of convincing support for the idea that interhemispheric interaction 147 underlies the bilateral saccade effects, more recent work has focused on the idea that saccade 
148 execution enhances cognition by altering attentional control. In one study, performance on the

149 well-established revised attentional network test (Fan et al. 2009) was assessed after either

150 repetitive bilateral saccades or central fixation (Edlin \& Lyle 2013). Saccade execution increased

151 the executive function network, which encompasses attentional control, by decreasing response

152 times to target stimuli in presence of response-incongruent flankers. In this study, the saccade-

153 induced enhancement of attentional control occurred independently of handedness consistency.

154 This raises the possibility that there could be a larger role for top-down attention when memories

155 are more difficult to access, and recent results support that saccade execution has a greater

156 facilitative effect on retrieval when recall and recognition are more difficult (Lyle \& Edlin 2015).

Outstanding Questions and Current Objectives

There remain several questions about the behavioral effects of repetitive saccades that have

160

not yet been explored. The first is how do repetitive saccades affect memory for complex novel

161 visual stimuli? The second involves how saccades affect stages of memory processing other than

162 retrieval. Most previous investigations have had subjects perform bilateral saccades after encoding

163 but before retrieval. It is not well understood how bilateral saccades affect the encoding of complex

164 novel visual stimuli, nor is it understood how these effects are modulated by handedness.

165 Understanding whether eye movements and handedness influence working memory or long-term

166 recognition memory or both is also relevant to a decades-long theoretical debate concerning

167 potential relationships between working memory and long-term memory. "Buffer" accounts (e.g.,

168 Baddeley's multi-component model) argue for a central executive coordinating separate

169 phonological and visuo-spatial short-term stores that are separate from long-term memory

170 (Baddeley 2003), while the hierarchically arranged embedded process account argues working 
171 memory is a subset of activated memory in the focus of attention and the subset of long-term

172 memory that is currently activated (Cowan 1999). To address these questions, we conducted an

173 exploratory study of the effects of repetitive bilateral saccades and handedness on scene memory.

174 We utilized a variant of the well-known Sternberg working memory paradigm (Sternberg 1966) in

175 which during a series of trials five pre-experimentally novel scenes are presented and held online

176 during a short $6 \mathrm{sec}$ delay period. After a 10-minute period of awake rest, we tested subjects' long-

177 term recognition memory for scenes previously maintained in short-term working memory. We

178 have shown previously that subjects perform this type of working memory task with scenes as

179 stimuli and can recognize far above chance dozens of scenes shown during the working memory

180 task after a long-term retention interval (Ellmore et al. 2015). Recent source analysis of scalp EEG

181 acquired with this task has implicated a right hemisphere parieto-occipital region that is active

182 when maintaining scenes in working memory, with temporal-spectral delay activity that correlates

183 with subsequent probe memory retrieval (Ellmore et al. 2017).

184 The specific objective of the present study was to test alternative hypotheses that making

185 repetitive saccades before or after encoding novel scenes in working memory would have either

186 beneficial or detrimental effects on subsequent memory as a function of handedness. A between-

187 subjects design was employed that allowed us to test 1) whether making repetitive saccades before

188 working memory encoding modulates subsequent working and long-term memory retrieval as a

189 function of handedness and 2) whether making repetitive saccades after working memory

190 encoding and before recognition modulates subsequent long-term recognition as a function of

191 handedness.

195 Subjects 
197 A total of 111 subjects $(M=20.74, S D=3.91$, range 18 to $40 ; 68$ females, 43 males $)$ were recruited

198 through the Sona Systems online experimental scheduling system of the City College of New York

199 Psychology Department. Each provided written informed consent, completed a revised Edinburgh

200 Handedness Inventory (EHI) (Williams 2010), and completed study procedures according to a

201 protocol approved (approval number 2015-0550) by the Institutional Review Board of the City

202 College of New York Human Research Protection Program. At the end of the study, each subject

203 received extra course credit for a total of one hour of participation.

204 The target sample size of over 100 subjects was determined based on similar sample sizes

205 used in previous published studies of eye movements and memory as well as on our ability to

206 recruit as many participants as possible during the date range for study recruitment which began

207 September 16, 2015 and ended November 22, 2017. In addition, for this study all measures,

208 conditions, and data exclusions are reported herein.

209

210

211

212

213

214

215

216

217

218

219

220

\section{Experimental Design}

All subjects completed a working memory task, followed by a 10-minute period of quiet awake rest, followed lastly by a test of recognition memory. The study utilized a between-subjects design

to manipulate eye movements by performance of two different eye tasks (saccade or fixation) executed either before performance of the working memory task (encoding group) or after the working memory task but before a test of recognition memory (recognition group). There was a total of 65 subjects $(M=20.86, S D=3.63$, range 18 to $38 ; 41$ females, 24 males $)$ in the encoding group and a total of 46 subjects $(M=20.56, S D=4.30$, range 18 to $40 ; 27$ females, 19 males $)$ in the recognition group. Within these two groups, subjects were assigned to perform either the saccade or fixation task using a pre-determined randomization schedule to minimize experimenter bias. 
Apparatus

All subjects completed tasks inside a closed room to minimize auditory and visual distractions.

Tasks were programmed in SuperLab 5 (Cedrus Corporation). Tasks were displayed on a 28-inch

LED monitor with a refresh rate of 60 Hertz and a screen resolution of 1920 by 1080 pixels.

Participants sat in the dark $96 \mathrm{~cm}$ from the monitor on an adjustable stool and positioned their head

228 in an Ultra Precision Head Positioner ${ }^{\mathrm{TM}}$ (Arrington Research, Inc), a combined chin and forehead rest, to help minimize head movements. The experimenter remained in the room for the duration of the experiment, but was seated outside of subjects' view. A black curtain separated the experimenter from the subject to minimize distraction. To ensure subjects complied with instructions when required to make saccades or fixation, an infrared light emitter and a $220 \mathrm{~Hz}$ eye-tracking camera (ViewPoint EyeTracker ${ }^{\circledR}$ by Arrington Research, Inc, www.ArringtonResearch.com) was placed between the monitor and the subject to track the pupil of one eye. The experimenter monitored the $\mathrm{x}$ and $\mathrm{y}$ eye position as measured by real-time computation of pupil center-of-mass on a separate monitor only visible to the experimenter. Subject behavioral responses were collected using a Cedrus RB-740 response pad placed on the table in front of them.

\section{Tasks}

Working Memory

Subjects completed 40 trials of a variant of a Sternberg working memory task (Sternberg 1966) used in previous experiments (Ellmore et al. 2015; Ellmore et al. 2017). Each trial consisted of a fixed load of 5 scene stimuli. A total of 220 pre-experimentally novel scenes were shown across the 40 trials of the working memory task. Each was a 24-bit color image of an outdoor scene 
248 sampled from the SUN database (Xiao et al. 2010). Scene stimuli were 800 by 600 pixels and were 249 presented at the center of the monitor subtending a visual angle of 16 by 12 degrees. On each trial,

250 five scenes appeared sequentially for $2 \mathrm{sec}$ each, followed by a $6 \mathrm{sec}$ delay period consisting of a

251 white screen with a foveal crosshair, during which subjects were required to keep these scenes in 252 mind, and then a probe scene was displayed for $2 \mathrm{sec}$ (Fig. 1a). Each trial was separated by $5 \mathrm{sec}$ 253 of black screen. Half (20) of the trials contained a positive probe, which was one of the five scenes 254 presented before the delay period. The other 20 trials contained a negative probe, a new scene not 255 previously shown in the set of scenes presented before the delay period. Positive probes were 256 randomly selected from each trial's five encoding stimuli so that the serial position of the probe 257 was equally likely to come from encoding position $1,2,3,4$, or 5 across the set of WM trials. 258 Positive and negative probe trials were randomly distributed across the set of 40 working memory 259 trials. Upon the presentation of the probe in each working memory trial, subjects were instructed 260 to press a green button on the response pad if the probe scene matched one of the scenes presented 261 in the set of 5 scenes shown before the 6 sec delay period; subjects were instructed to press a red

262 button if the probe scene did not match one of the scenes presented in the set of 5 scenes shown 263 before the delay. For each working memory probe presentation, subjects were required to respond 264 within the 2 seconds that the probe was on the screen. Before performing the working memory 265 task, subjects were given a 5-minute demonstration task with different stimuli (animals) to ensure 266 that they understood task instructions before the actual working memory task began.

267

268

269

270

271

272

\section{Recognition Memory}

After completing the working memory task, subjects were allowed to disengage from the head restraint and rest quietly in the experiment room for ten minutes. At the end of this ten-minute retention interval, subjects completed a task of recognition memory (Fig. 1b) that included a total 
273 of 200 scenes. One hundred of these scenes were randomly sampled from the sets of scenes

274 presented before the delay in each of the 40 previous working memory trials. A total of 8 out of

275 these 100 "old" stimuli were previously used as positive probes in the WM task. The other 100

276 scenes were completely new scenes sampled randomly from the SUN database. The 100 old and

277100 new scenes were presented one at a time for $2 \mathrm{sec}$ each in random order as individual trials.

278 For each scene presentation during the recognition test, subjects were required to respond within

279 the 2 seconds that the probe was on the screen. A 2 sec black screen separated each trial. For each

280 trial, subjects were instructed to press the green button on the response pad to indicate that they

281 recognized having previously viewed the scene; subjects were instructed to press the red button to

282 indicate that they did not recognize the scene.

283

284

285

286

287

288

289

290

291

292

293

294

295

296

297

\section{[insert Figure 1 here]}

\section{Eye Tasks}

The main experimental manipulation required subjects to perform one of two eye tasks (saccade or fixation) either before the working memory task or before the recognition memory task. The saccade task required subjects to make deliberate repetitive horizontal saccades for $30 \mathrm{sec}$. Each horizontal saccade was cued by the appearance of a white disc on a black background. The diameter of the white disk was 70 pixels (1.49 degrees of visual angle). Each dot appeared for 500 $\mathrm{ms}$ and alternated its position between the right and left sides of the screen for a total of $30 \mathrm{sec}$ (Fig. 2a). The center-to-center distance of the disk as it alternated between the right and left sides of the screen was 1285 pixels (27.3 degrees of visual angle). The fixation task required that subjects fixate a single disc (same size as the white disc in the saccade task) that was stationary at the center of the screen. The disc appeared on a black background for $30 \mathrm{sec}$ and cycled through 6 colors 
300 (red, blue, yellow, green, pink, and purple), with each color presented individually for $500 \mathrm{~ms}$ (Fig.

301 2b). To keep subjects' attention on the different eye tasks, subjects were instructed to count

302 covertly the number of times the disc alternated positions between the left and right screen

303 positions; during the fixation task, subjects were instructed to count covertly the number of times

304 the disc changed colors. The number of disc alternations and color changes was equal.

305

306

307

308

309

310

311

312

313

314

315

316

317

318

319

320

321

322

323

324

325

326

[insert Figure 2 here]

\section{Analysis}

The analysis began with the computation of a laterality quotient (LQ) from the responses to the eight questions of the EHI-R (Williams 2010). The EHI-R (https://doi.org/10.13140/RG.2.2.33298.25284) is similar to the original EHI published in 1971 (Oldfield 1971). Highly variable usage of modified versions of the original EHI are prominent in the scientific literature, which may imperil efforts to produce replicable and convergent findings (Edlin et al. 2015). Therefore, it is important to explain and scientifically justify differences in any revision and note differences with the inventory used in many other labs (Lyle et al. 2008b). Compared to the original EHI, the EHI-R discards three items ("broom", "opening box", and "drawing") and adds a new one - "computer mouse" - making the total number of items eight. It also uses the Likert format in an attempt to simplify the confusing instructions of the original EHI. The items "opening box" and "broom" are removed based on statistical evidence including factor analytic studies (Dragovic 2004; Williams 1986) that find a single Handedness factor whose loadings on it of these two activities are low outliers. "Drawing" is removed because it could be used as a substitute for writing since drawing and writing are very highly (about 0.9 ) correlated and the inclusion of both adds little new information. The addition of "computer mouse" is based 
327 on a suggestion by Dragovic (Dragovic 2004). The use of a computer mouse is one major

328 unimanual activity in today's world that was not widespread forty years ago when the EHI was

329 developed. Additionally, the EHI-R has been used in several recent studies (Eriksen et al. 2018;

330 Holcombe et al. 2017; Kielar et al. 2016; Rice \& M. Gosser 2015). The EHI-R asks subject to

331 indicate which hand they use to complete various everyday activities (e.g., striking a match, using

332 a computer mouse). Participants indicated their handedness preference for each item by checking

333 off one of the following response options (with corresponding point values): "always right" (50)

334 "usually right" (25), "no preference" (0), "usually left" (-25), and "always left (-50). The LQ was

335 calculated by tallying the point values for all eight items and dividing the total score by four,

336 resulting in LQ scores that ranged from +100 (right handed) to -100 (left handed). A total of 7

337 subjects scored as left-handed $(\mathrm{LQ}<0)$, one subject scored perfectly mixed $(\mathrm{LQ}=0), 33$ subjects

338 scored non-strongly right-handed $(\mathrm{LQ}<80)$, and 78 subjects scored strongly-right handed

$339(\mathrm{LQ}>=80)$.

340 The next step in the analysis was to compute for each subject performance expressed as

341 overall percentage correct and sensitivity as d-prime for the 40 trials of the working memory task

342 and separately for the 200 trials of the recognition memory task. The d-prime sensitivity measure

343 from signal detection theory accounts for effects of response bias (Stanislaw \& Todorov 1999).

344 The proportions of total hits and false alarms were first calculated for each subject and each task.

345 A hit was counted when a previously presented stimulus (i.e., a positive probe in the working

346 memory task) was signaled by the subject pressing a button indicating, correctly, that the stimulus

347 had been previously seen (an old stimulus correctly classified as old). A false alarm was counted

348 when a (new) stimulus not previously presented (i.e., a negative probe in the working memory

349 task) was indicated by the subject pressing a button indicating, incorrectly, that the stimulus had 
350 been previously presented (a new stimulus incorrectly classified as an old stimulus). Then d-prime

351 was computed as the difference in standardized normal deviates of hits minus false alarms: $Z$ (hit

352 rate $)-Z($ false alarm rate). Separate linear regression analyses were then performed with the

353 criterion variables (Y) percent correct accuracy and d-prime sensitivity. The predictor variables

354 (X) were raw LQ for direction of handedness and the absolute values of LQ for degree of

355 handedness. Regressions were computed to predict the criterion variables of accuracy or d-prime

356 by either direction or degree of handedness as a function of the eye-movement task (fixation vs.

357 saccade) and placement of the eye-movement task (before the working memory or recognition

358 memory task). Regression analyses were computed in GraphPad Prism version 7.0 (GraphPad

359 Software, La Jolla California USA, www.graphpad.com). Statistical tests of the regression slopes

360 were also computed to assess differences in performance as a function of laterality and type and

361 placement of the eye-movement task. Statistical analysis of regression intercepts (elevations) was

362 used to assess differences in overall performance as a function of type and placement of the eye-

363 movement task. The raw data input to these analyses is provided in the Supplemental File.

364

365

366

367

368

369

370

371

372

373

374

375

376

\section{Results}

Working Memory Performance

Analysis of Regression Slopes

Linear regression was used to quantify the relationship between working memory performance, as measured by both percent correct accuracy and d-prime, and direction of laterality as a function of the eye task. The statistical tests assessed whether the slope of the regression equation differed significantly from zero. Similar tests were used to assess the relationship between the dependent measures and the degree of laterality, indicating consistency of handedness, using the absolute value of the laterality quotient. 

accuracy increased non-significantly as a function of direction of laterality (slope $=0.03098 \pm$ 0.03765 SE, $F_{(1,31)}=0.6774, p=0.4168$, Fig 3a blue line and blue circles). The d-prime measure also increased non-significantly as a function of direction of laterality (slope $=0.00168 \pm 0.00337$ $\mathrm{SE}, \mathrm{F}_{(1,31)}=0.2473$, $\mathrm{p}=0.6225$, Fig $3 \mathrm{~b}$ blue line and blue circles). Working memory percent correct accuracy increased non-significantly as a function of degree of laterality (slope $=0.02432 \pm 0.05178$ increased non-significantly as a function of degree of laterality (slope $=0.001734 \pm 0.00461 \mathrm{SE}$, $\mathrm{F}_{(1,31)}=0.141, \mathrm{p}=0.7098$, Fig $3 \mathrm{~d}$ blue line and blue circles $)$.

When saccades occurred before the working memory task, working memory percent correct accuracy increased significantly as a function of direction of laterality (slope $=0.1029 \pm$ increased non-significantly as a function of direction of laterality (slope $=0.0029 \pm 0.0032 \mathrm{SE}$,

$\mathrm{SE}, \mathrm{F}_{(1,30)}=0.694, \mathrm{p}=0.4114$, Fig $3 \mathrm{c}$ red line and red triangles). The d-prime measure also increased non-significantly as a function of degree of laterality (slope $=0.01137 \pm 0.00984 \mathrm{SE}, \mathrm{F}_{(1,30)}=1.334$, $\mathrm{p}=0.2571$, Fig $3 \mathrm{~d}$ red line and red triangles). a control group, as one would expect no influence of these eye tasks on working memory

397 performance if they occur after the working memory task. Consistent with this prediction, when

398 fixation occurred after the working memory task, working memory percent correct accuracy 399 increased non-significantly as a function of direction of laterality (slope $=0.02973 \pm 0.02872 \mathrm{SE}$, 
$400 \mathrm{~F}_{(1,21)}=1.072, \mathrm{p}=0.3123$, Fig 3a grey line and grey boxes). The d-prime measure also increased

401 non-significantly as a function of direction of laterality (slope $=0.00116 \pm 0.00268$ SE,

$402 \mathrm{~F}_{(1,21)}=0.1873, \mathrm{p}=0.6696$, Fig $3 \mathrm{~b}$ grey line and grey boxes). Working memory percent correct

403 accuracy increased non-significantly as a function of degree of laterality (slope $=0.06811 \pm 0.05039$

$404 \mathrm{SE}, \mathrm{F}_{(1,21)}=1.827, \mathrm{p}=0.1909$, Fig $3 \mathrm{c}$ grey line and grey boxes). The d-prime measure also increased

405 non-significantly as a function of degree of laterality (slope $=0.00620 \pm 0.00462 \mathrm{SE}, \mathrm{F}_{(1,21)}=1.805$,

$406 \mathrm{p}=0.1935$, Fig $3 \mathrm{~d}$ grey line and grey boxes).

407

When saccades occurred after the working memory task, working memory percent correct 408 accuracy increased non-significantly as a function of direction of laterality (slope $=0.005429 \pm$ 409 $0.02251 \mathrm{SE}, \mathrm{F}_{(1,21)}=0.05818, \mathrm{p}=0.8117$, Fig $3 \mathrm{a}$ cyan line and cyan stars). The d-prime measure

410 decreased non-significantly as a function of direction of laterality (slope=-0.00034 $\pm 0.0025 \mathrm{SE}$,

$411 \mathrm{~F}_{(1,21)}=0.0187, \mathrm{p}=0.8926$, Fig $3 \mathrm{~b}$ cyan line and cyan stars). Working memory percent correct

412 accuracy increased non-significantly as a function of degree of laterality (slope $=0.0035 \pm 0.03747$

$413 \mathrm{SE}, \mathrm{F}_{(1,21)}=0.0087, \mathrm{p}=0.9265$, Fig $3 \mathrm{c}$ cyan line and cyan stars $)$. The d-prime measure also increased

414 non-significantly as a function of degree of laterality (slope=-6.445e-005 $\pm 0.00422 \mathrm{SE}$, $415 \mathrm{~F}_{(1,21)}=0.00023, \mathrm{p}=0.9880$, Fig $3 \mathrm{~d}$ cyan line and cyan stars).

416

417

418

419

420

421

422

423

424

\section{Analysis of Regression Intercepts}

While analysis of regression slopes indicates change in performance as a function of direction or degree of laterality and eye task timing, evaluation of the regression intercepts (i.e., elevations) indicates whether the overall levels of performance differ.

When considering relationships of performance with direction of laterality, the four intercepts of Fig 3a were significantly non-zero $\left(\mathrm{F}_{(3,106)}=6.164, \mathrm{p}=0.0007\right)$ with best working memory percent correct performance when saccades were made after the working memory task 
$425(93.28 \% \pm 1.844$ SE, 95\% C.I.: 89.45 to 97.12$)$ and worst working memory percent correct 426 performance when saccades were made before the working memory task $(77.79 \% \pm 3.84,95 \%$ 427 C.I.: 69.95 to $85.64 \%$ ). The four intercepts of Fig $3 \mathrm{~b}$ were also significantly non-zero $428\left(\mathrm{~F}_{(3,106)}=3.742, \mathrm{p}=0.0133\right)$ with best working memory d-prime occurring when saccades were made 429 after the working memory task (3.179 \pm 0.2082 SE, 95\% C.I.: 2.746 to 3.612) and worst working 430 memory d-prime when saccades were made before the working memory task $(2.442 \pm 0.2819$, $43195 \%$ C.I.: 1.847 to 2.998$)$. When considering relationships of performance with degree of laterality, the four intercepts 433 of Fig $3 \mathrm{c}$ were significantly non-zero $\left(\mathrm{F}_{(3,106)}=5.699, \mathrm{p}=0.0012\right)$ with best working memory percent correct performance when saccades were made after the working memory task $(93.38 \% \pm 3.07 \mathrm{SE}$, 95\% C.I.: 87.00 to 99.77 ) and worst working memory percent correct performance when saccades were performed before the working memory task $(75.2 \% \pm 12.80,95 \%$ C.I.: 49.05 to $101.30 \%)$.

437 The four intercepts of Fig $3 d$ were also significantly non-zero $\left(\mathrm{F}_{(3,106)}=3.909, \mathrm{p}=0.0108\right)$ with best 438 working memory d-prime occurring when saccades were made after the working memory task $(3.15 \pm 0.3463$ SE, 95\% C.I.: 2.43 to 3.87$)$ and worst working memory d-prime when saccades were made before the working memory task $(1.658 \pm 0.866,95 \%$ C.I.: -0.1106 to 3.427$)$.

[insert Figure 3 here]

444

445

446

\section{Recognition Performance}

\section{Analysis of Regression Slopes}

Linear regression was also used to quantify the relationship between recognition memory, as

451 measured by both percent correct accuracy and d-prime, and direction of laterality as a function of

452 the eye task. The statistical test assessed whether the slope of the regression equation differed 
453 significantly from zero. Similar tests were used to assess the relationship between the dependent 454 measures and the degree of laterality, indicating consistency of handedness, using the absolute 455 value of the laterality quotient.

456 When fixation occurred before the working memory task, later recognition percent correct 457 accuracy increased non-significantly as a function of direction of laterality (slope $=0.03746 \pm$ 4580.04449 SE, $F_{(1,31)}=0.7091, p=0.4062$, Fig $4 a$ blue line and blue circles). The d-prime measure also 459 increased non-significantly as a function of direction of laterality (slope $=0.00208 \pm 0.00281 \mathrm{SE}$, $460 \mathrm{~F}_{(1,31)}=1.351, \mathrm{p}=0.2542$, Fig $4 \mathrm{~b}$ blue line and blue circles). Recognition memory percent correct 461 accuracy increased non-significantly as a function of degree of laterality (slope $=0.03984 \pm 0.06102$ 462 SE, $F_{(1,31)}=0.4263, p=0.5186$, Fig $4 \mathrm{c}$ blue line and blue circles). The d-prime measure also 463 increased non-significantly as a function of degree of laterality (slope $=0.00344 \pm 0.00383 \mathrm{SE}$, $464 \mathrm{~F}_{(1,31)}=0.811, \mathrm{p}=0.3747$, Fig $4 \mathrm{~d}$ blue line and blue circles).

465 When saccades occurred before the working memory task, later recognition percent correct 466 accuracy increased non-significantly as a function of direction of laterality (slope $=0.06502 \pm$ $4670.04615 \mathrm{SE}, \mathrm{F}_{(1,30)}=1.985, \mathrm{p}=0.1691$, Fig 4a red line and red triangles). The d-prime measure for 468 later recognition also increased non-significantly as a function of direction of laterality 469 (slope $=0.0032 \pm 0.0027 \mathrm{SE}, \mathrm{F}_{(1,30)}=1.351, \mathrm{p}=0.2542$, Fig $4 \mathrm{~b}$ red line and red triangles). 470 Recognition memory percent correct accuracy also increased non-significantly as a function of 471 degree of laterality (slope $=0.0629 \pm 0.1472 \mathrm{SE}, \mathrm{F}_{(1,30)}=0.1829, \mathrm{p}=0.6720$, Fig $4 \mathrm{c}$ red line and red 472 triangles). The d-prime measure for later recognition also increased non-significantly as a function 473 of degree of laterality (slope $=0.0043 \pm 0.00863 \mathrm{SE}, \mathrm{F}_{(1,30)}=0.2443, \mathrm{p}=0.6247$, Fig $4 \mathrm{~d}$ red line and 474 red triangles). 

task, recognition percent correct accuracy increased significantly as a function of direction of

477 laterality $\left(\right.$ slope $=0.08738 \pm 0.03277 \mathrm{SE}, \mathrm{F}_{(1,21)}=7.111, \mathrm{p}=0.0144$, Fig 4 a grey line and grey boxes).

478 The d-prime measure for recognition increased marginally significantly as a function of direction 479 of laterality (slope $=0.00507 \pm 0.00247 \mathrm{SE}, \mathrm{F}_{(1,21)}=4.23, \mathrm{p}=0.0523$, Fig $4 \mathrm{~b}$ grey line and grey 480 boxes). Recognition memory percent correct accuracy increased non-significantly as a function of 481 degree of laterality ( lope $=0.1014 \pm 0.0639 \mathrm{SE}, \mathrm{F}_{(1,30)}=2.517, \mathrm{p}=0.1276$, Fig $4 \mathrm{c}$ grey line and grey 482 triangles). The d-prime measure for recognition also increased non-significantly as a function of 483 degree of laterality (slope $=0.00714 \pm 0.00456 \mathrm{SE}, \mathrm{F}_{(1,30)}=2.449, \mathrm{p}=0.1326$, Fig $4 \mathrm{~d}$ grey line and 484 grey triangles).

485 When saccades occurred after the working memory task and before the recognition 486 memory task, recognition percent correct accuracy increased non-significantly as a function of 487 laterality $\left(\right.$ slope $=0.03398 \pm 0.03797 \mathrm{SE}, \mathrm{F}_{(1,21)}=0.8009, \mathrm{p}=0.3810$, Fig 4 a cyan line and cyan stars). 488 The d-prime measure for recognition increased non-significantly as a function of direction of 489 laterality (slope $=0.0028 \pm 0.00253 \mathrm{SE}, \mathrm{F}_{(1,21)}=1.274, \mathrm{p}=0.2718$, Fig $4 \mathrm{~b}$ cyan line and cyan stars). 490 Recognition memory percent correct accuracy increased non-significantly as a function of degree 491 of laterality (slope $=0.0679 \pm 0.0626 \mathrm{SE}, \mathrm{F}_{(1,30)}=1.18, \mathrm{p}=0.2897$, Fig $4 \mathrm{c}$ cyan line and cyan stars).

492 The d-prime measure for recognition also increased non-significantly as a function of degree of 493 laterality (slope $=1.115 \pm 0.3462 \mathrm{SE}, \mathrm{F}_{(1,30)}=1.076, \mathrm{p}=0.3113$, Fig $4 \mathrm{~d}$ cyan line and cyan stars).

494

495

496

497

498

\section{Analysis of Regression Intercepts}

Analysis of regression intercepts was conducted to determine whether the overall level of recognition performance differed as a function of the eye task timing. The four intercepts of Fig 4a representing recognition percent correct accuracy versus direction of laterality were 
500 significantly non-zero $\left(\mathrm{F}_{(3,106)}=4.721, \mathrm{p}=0.0039\right)$ with best recognition memory performance for

501 the condition of fixation occurring before the working memory task $(70.08 \% \pm 3.899$ SE, $95 \%$

502 C.I.: 62.12 to $78.03 \%$, Fig 4 a blue line and blue circles) and the lowest recognition memory

503 performance in the condition of saccades occurring before the working memory task $(60.67 \% \pm$

5044.06 , 95\% C.I.: 52.38 to $68.96 \%$, Fig 4 a red line and red triangles). Recognition performance

505 during the condition when saccades were made before the recognition memory task $(70.06 \% \pm$

$5063.111,95 \%$ C.I.: 63.59 to 76.53 , Fig 4a cyan line and cyan stars) was greater than performance

507 during the condition of fixation before the recognition memory task $(64.75 \% \pm 2.819,95 \%$ C.I.:

50858.38 to $70.62 \%$, Fig 4 a grey line and grey boxes), but the difference was not significantly different

509 (two-tailed $\mathrm{t}_{(44)}=1.2648, \mathrm{p}=0.2126$, Cohen's $\mathrm{d}=0.3729$ ). The level of recognition performance in

510 the condition when saccades were made before the recognition task was nearly identical to

511 performance after the condition of fixation before the working memory task $(70.08 \% \pm 3.899,95 \%$

512 C.I.: 62.12 to $78.03 \%$, Fig 4a blue line and blue circles). These virtually identical levels of 513 performance make the blue and cyan lines of Fig 4a appear to overlap.

514 The four intercepts of Fig $4 \mathrm{~b}$ representing d-prime versus direction of laterality were

515 significantly non-zero $\left(\mathrm{F}_{(3,106)}=3.964, \mathrm{p}=0.0101\right)$ with best recognition memory performance for

516 the condition of fixation occurring before the working memory task $(1.327 \pm 0.2467$ SE, $95 \%$ C.I.:

5170.8235 to 1.83 , Fig $4 \mathrm{~b}$ blue line and blue circles) and the lowest recognition memory performance

518 in the condition of saccades occurring before the working memory task $(0.8231 \pm 0.2408,95 \%$

519 C.I.: 0.3313 to 1.315 , Fig $4 \mathrm{~b}$ red line and red triangles).

520 The four intercepts of Fig $4 \mathrm{c}$ representing recognition percent correct accuracy versus

521 degree of laterality were significantly non-zero $\left(\mathrm{F}_{(3,106)}=4.863, \mathrm{p}=0.0033\right)$ with best recognition

522 memory performance for the condition of fixation occurring before the working memory task 
$523(69.76 \% \pm 5.348$ SE, 95\% C.I.: 58.86 to 80.67 , Fig 4c blue line and blue circles) and the lowest

524 recognition memory performance in the condition of saccades occurring before the working

525 memory task $(60.22 \pm 12.95$, 95\% C.I.: 33.78 to 86.66 , Fig $4 \mathrm{c}$ red line and red triangles).

526 The four intercepts of Fig 4d representing recognition d-prime versus degree of laterality

527 were significantly non-zero $\left(\mathrm{F}_{(3,106)}=4.235, \mathrm{p}=0.0072\right)$ with best recognition memory performance

528 for the condition of fixation occurring before the working memory task $(1.205 \pm 0.3354 \mathrm{SE}, 95 \%$

529 C.I.: 0.5211 to 1.889 , Fig $4 d$ blue line and blue circles) and the lowest recognition memory

530 performance in the condition of saccades occurring before the working memory task $(0.6978 \pm$

5310.7593 , 95\% C.I.: -0.853 to 2.249 , Fig $4 d$ red line and red triangles).

532

533

534

535

536

537

538

539

[insert Figure 4 here]

\section{Discussion}

Humans have a remarkable ability to remember a large number of scenes and other complex visual

540 stimuli even when given only a short period of time to encode them (Brown \& Scott 1971; Shepard

541 1967; Standing 1973; Standing et al. 1970). Scene memory is also an important component of

542 episodic autobiographical memory. Normal as well as disordered reminiscence often includes

543 vivid mental recollection of scenes where past events took place (Brewin 2014; Burgess et al.

544 2002). In addition, recognition of a previously encountered scene in, for example an old

545 photograph, is often quick, highly accurate, and can trigger episodic memory retrieval. Therefore,

546 it is important to understand how scene memory is influenced by multifactorial traits like

547 handedness and by simple behavioral manipulations (e.g., bilateral saccades) shown previously to 548 influence episodic memory retrieval. 
550 memory is modulated by handedness and by bilateral horizontal saccades made before scene 551 encoding and recognition. Previous studies have shown that strongly right-handed and 552 consistently-handed benefit most from bilateral saccades (Chu et al. 2012; Lyle et al. 2012; Lyle 553 \& Orsborn 2011; Prichard \& Christman 2017; Propper et al. 2017), but these studies focused on 554 mostly verbal aspects of explicit or episodic memory retrieval and had subjects make the saccades 555 after encoding and before retrieval. The two novel aspects of the present study include, first, the 556 focus on visual scene memory and, second, having different groups of subjects make saccades 557 before encoding as well as before recognition.

558 There are two main findings of the present study. First, when saccades were made before 559 encoding scenes in working memory, performance as measured by regression slopes relating 560 overall percent correct with direction of handedness increased significantly positively (Fig 3a) with 561 right-handers (+LQ) performing better than left-handers (-LQ). Regression slopes exhibited the 562 same trend of increasing magnitude when a d-prime measure of sensitivity was related to raw LQ 563 scores (Fig 3b), but there was no significant relationship suggesting some effects of response bias 564 may have been present in the subjects when a measure of overall percent correct accuracy was used. Regression slopes were mostly positive when percent correct and d-prime were regressed against absolute LQ, a measure of degree or consistency of handedness, but these tests also did not reach significance. Performance also did not correlate significantly as a function of direction of handedness when either the saccade or fixation condition came after the scene working memory task.

The second main result was that there was no apparent benefit of direction or degree of

571 handedness on making saccades before scene recognition. Recognition performance in the group 
572 of subjects who made saccades after working memory and before recognition increased non-

573 significantly as a function of both direction and degree of handedness. Recognition performance

574 as measured by both overall percent correct and d-prime in the group of subjects who fixated after

575 working memory and before recognition did increase significantly as a function of direction of

576 handedness, with better performance as LQ increased indicating an advantage for right-handed

577 subjects. The relationships between recognition performance and degree of handedness in those

578 who fixated after working memory and before recognition were non-significantly positive.

579 Finally, the subjects who made saccades before the working memory task performed the poorest

580 during a subsequent test of recognition memory suggesting a link between poor encoding in

581 working memory and poor subsequent long-term recognition memory.

582 Why would making saccades before encoding scenes in working memory result in better

583 performance for strongly right-handed (+LQ) but not necessarily for consistent-handed

584 individuals? There is some neuroimaging evidence suggesting that form-specific perceptual

585 aspects of scene encoding may be right lateralized in the brain (Brewer et al. 1998; Stevens et al.

586 2012), with form-abstract and language-specific aspects including the verbalizability of the visual

587 stimuli lateralized to the left (Golby et al. 2001; Stevens et al. 2012). Our recent source analysis

588 of working memory delay period EEG implicates right parieto-occipital focus of activity that

589 covaries positively as a function of performance. Due to limited sample size in that study, it's not

590 possible to draw conclusions about whether mixed- or left-handers have a more symmetrical

591 pattern of activity during scene encoding and memory maintenance that correlates with worse

592 performance. However, a tentative explanation is that strongly right-handed individuals may have

593 an advantage over mixed- and left-handers in controlling spatial attention for scene encoding and

594 memory. Right handers perform better than either mixed or left handers on tasks requiring 
595 reproduction from memory of some aspects of geometric visual material, while left and mixed

596 handers do not differ from one another (Nebes \& Briggs 1974). A hemispheric advantage for

597 directing spatial attention could protect these right-handers from potentially destabilizing effects

598 of repetitive saccades on visuospatial attention processes needed to encode the perceptual aspects

599 of scenes. While saccades may be beneficial to mixed/inconsistent-handers before the retrieval of

600 explicit episodic memories by improving attentional control - a form of top-down inward control

601 - they may be detrimental to control of visuospatial attention directed outward to maintain a stable

602 reference frame for encoding details of complex visual information contained in scenes. Fitting

603 with this idea, there is evidence for hemispheric specialization at early levels of visual analysis to

604 the right for processing low spatial frequencies and to the left for processing high spatial

605 frequencies, with supplementary activation of right inferior parietal lobule reflecting attentional

606 modulation (Peyrin et al. 2004).

607 A growing number of studies have demonstrated saccade induced benefits specifically on 608 recognition (Brunye et al. 2009; Lyle \& Orsborn 2011; Parker \& Dagnall 2007; Parker et al. 2008).

609 In the present study, when bilateral saccades were made before scene recognition but not before

610 scene working memory, we found a small but not statistically significant increase in performance

611 relative to simple fixation. Performance was best for consistent right-handers, which includes those

612 who have an LQ near +100 in Fig 3 and Fig 4 because they indicate they perform all tasks "always"

613 with their right hand. A finding reported recently is that beneficial effects of pre-task saccades

614 occur in consistent-handed individuals (Lyle et al. 2012), those who perform most actions 615 consistently with either their right or their left hand. If this pattern was present in our data, we 616 might see an inverted U-shaped distribution of data points in Fig 4 with lower baseline 617 performance for both -LQ (consistent left-handers) and +LQ (consistent right-handers) relative to 
618 mixed-handers. The ends of the inverted U-shaped might then be shifted up and/or flattened

619 relative to fixation following saccades. Benefits are not seen in Fig 4 for both left and right

620 consistent-handed individuals. Rather, it appears that only consistent right-handers are better able

621 to deal with the detrimental effects of bilateral saccades made before scene working memory (as

622 indicated by the significant slope of the red line in Fig 3a). Consistent right-handers also have

623 better recognition memory in general because when they fixate before the recognition test they

624 perform better (as indicated by the significant slope of the grey line in Fig 4a).

The result of the present study represents a novel amendment to the important findings of

previous studies showing better overall memory in inconsistent handers and beneficial effects of saccades in consistent-handers. Those findings have replicated in more recent studies (Chu et al. 2012; Parker et al. 2017; Prichard \& Christman 2017; Propper et al. 2017). Many of the tasks used

to assess episodic and/or autobiographical memories operate in the verbal domain. In the present study, subjects might default to a familiarity-based and highly-visual implicit "know" strategy

631 rather than rely on a verbal episodic-like type of remembering. Adopting a familiarity-based strategy in the present experiments is even more likely given the speeded choices subjects were required to make within the $2 \mathrm{sec}$ stimulus viewing windows. Eye movement effects have been demonstrated to be more beneficial for remember/recollective responses or for tasks that demand recollection for accurate responses rather than familiarity (Lyle et al. 2012; Parker et al. 2008). In addition, many recognition tasks shown to benefit from bilateral saccades rely on word lists for

637 memoranda (Parker \& Dagnall 2007; Propper et al. 2017; Samara et al. 2011), content that is much

638 different from the novel scene stimuli used in the present study. However, it is also worth noting 639 that the beneficial effects of saccades do not manifest in implicit memory tasks even when those 640 tasks involve words (Christman et al. 2003) had participants study a short word list and then tested 
641 them on a word-stem completion task, consisting of an equal mix of new and previously seen

642 words. The findings showed that none of the eye movement conditions enhanced word fragment 643 completion performance compared to the fixation condition. This was interpreted to indicate that 644 saccades do not enhance implicit forms of memory that are likely reflective of intra-hemispheric 645 activity (Christman et al. 2003).

646 One recent study of saccade related enhancement is notable for its use of visual-spatial 647 stimuli and an exploration of whether effects could be obtained when eye movements were made 648 before encoding. Brunye et al. (Brunye et al. 2009) used an encoding task that lasted a total of only 649120 seconds and comprised a small number of just four stimuli, all aerial maps that were acquired 650 from satellite pictures. The authors found increased recognition sensitivity and decreased response 651 times with effects only seen when eye movements preceded episodic memory retrieval, but not 652 when they preceded encoding. The effects were strongest for strongly right-handed individuals. 653 On the basis of the HERA model (Nyberg et al. 1996), Brunye et al theorized that eye movements 654 would not benefit encoding due to unilateral activity, but would be beneficial with recognition 655 tests demanding a high degree of right and left-hemisphere activity.

656 The present study has some limitations. One limitation is the few strongly left-handed 657 subjects in our sample. Although we found that when saccades occurred before the working 658 memory task the WM percent correct accuracy increased significantly as a function of direction of 659 laterality, this result could have been driven by the single very low performing left-hander 660 represented by the red triangle in the lower left corner of Figure 3a. The few left-handed subjects 661 and large number of strongly-right handed subjects in the sample rules out a traditional analysis to 662 assess group-level differences. Instead in the present study relative performance differences as a 663 function of LQ were characterized using regression slopes. Therefore, summaries of results 
664 involving right-handers (+LQ) performing better than left-handers (-LQ) are not intended and

665 should not be taken to imply there were group-level differences between equal samples of left- and

666 right-handers. Another limitation is that the working memory task utilized a relatively high load

667 of 5 scene stimuli per trial and required a large number of scenes to be memorized and later

668 discriminated in the recognition test. The 200-trial recognition test took over thirteen minutes to

669 complete and it is possible that if there were any beneficial effects of preceding eye movements

670 they may have reduced across the lengthy time interval taken for subjects to complete the task.

671 This increased the overall difficulty of the memory tasks with performance during the working

672 memory task nowhere near ceiling. Prior studies involving saccade manipulation have used

673 relatively smaller numbers of items for encoding and have frequently required participants to view

674 stimuli passively during learning, rather than engage them in working memory. Both the limited

675 number of items and the nature of the encoding tasks used in previous research require a lower

676 degree of cognitive processing compared to the high-load working memory and recognition tasks

677 used here. Both tasks far exceeded the numbers of stimuli used in prior studies and could have

678 potentially decreased overall encoding and consolidation into long-term memory given the short

679 10-minute interval separating working memory and recognition testing. For the eye tasks, the

680 requirement for subjects to count disc alternations and color changes was to ensure subjects

681 performed the tasks. However, an important difference to acknowledge is that counting has not

682 commonly been used in previous studies of saccade effects on memory. While our sample included

683111 participants, there was an unequal sample of strongly left-handed compared to strongly-right

684 handed subjects making the degree of handedness (i.e., consistency comparisons) weighted

685 unequally with strongly-right handed subjects. Finally, the study design relied on between-subjects 
686 manipulation of conditions, which tends to increase variance and decrease statistical power relative

687 to a within-subject design.

688

689

690

691 We found evidence that performance of repetitive horizontal saccades before scene working and

692 recognition memory affects performance differently depending on the direction but not degree of

693 handedness. Making repetitive saccades before encoding scenes in working memory has

694 detrimental effects on both short- and long-term memory, with the most pronounced effects for

695 those who are not strongly right-handed. Moreover, saccade execution before scene recognition

696 does not appear to benefit recognition accuracy appreciably. Making repetitive saccades before

697 scene encoding may be detrimental because the eye movements destabilize subsequent

698 visuospatial processing. If working and long-term memory systems interact, then scenes that are

699 poorly encoded initially will not transfer to long-term memory. Right handers may have an

700 advantage in dealing with such disruption due to more lateralized mechanisms for scene

701 processing. These tentative explanations require confirmation by future experiments combining

702 both behavior and brain measurements.

703

704 Acknowledgements

705

706 The authors thank Iffat Noor and Chelsea Reichert for assistance with data collection. The content

707 is solely the responsibility of the authors and does not necessarily represent the official views of

708 the National Institutes of Health.

709

710 


\section{Figure Legends}

712

713 Figure 1. Example Scene Working and Recognition Memory Trials. Each working memory

714 trial (a) consisted of an encoding phase of five scene stimuli, each presented for 2 sec, followed

715 by a 6 sec delay period of crosshair fixation and a $2 \mathrm{sec}$ presentation of either a positive probe

716 (scene from the encoding set) or a negative probe (new scene). On each trial, there was a $50 \%$

717 chance of a positive probe appearing. Each recognition memory trial (b) consisted of alternating

718 presentation of a scene stimuli for $2 \mathrm{sec}$ followed by a black screen for $2 \mathrm{sec}$. Old and new scenes

719 were randomly intermixed. Subjects were instructed to press a green button if they had seen a

720 stimulus in any of the previous working memory trials and a red button if they had never seen the

721 stimulus before. In this example, image numbers from the set of scene stimuli are listed rather than

722 the actual scene images to illustrate how scenes during encoding could be presented as a positive

723 probe (a) or as old stimuli in the recognition task (b).

Figure 2. The Saccade and Fixation Eye Tasks. The saccade task (top left) required subjects to alternate for 30 seconds looking toward the left and right as a white disc moved back and forth across the screen. Example eye traces (top right) show saccadic movements with large periodic deviations in the x position (horizontal) trace with a stable y position (vertical) trace. The fixation task (bottom left) required subjects to maintain fixation on a center disc as it changed color. Example eye-tracking traces (bottom right) show fixation, with minimal deviations in the $\mathrm{x}$

731 (horizontal) and y position (vertical) traces.

732

733 Figure 3. Scene Working Memory Performance as a Function of Laterality and Eye Task

734 Timing. There was a significant relationship in working memory percent correct performance as 
735 a function of direction of laterality when saccades were made before the working memory task

736 (non-zero slope of $0.1029 \pm 0.04365 \mathrm{SE}, \mathrm{F}_{(1,30)}=5.56, \mathrm{p}=0.0251$, red line and red triangles, panel

737 a). Overall mean performance among conditions differed (non-zero intercepts, $F_{(3,106)}=6.164$,

$738 \mathrm{p}=0.0007)$ with lowest working memory percent correct performance obtained when saccades

739 were made before the working memory task $(77.79 \% \pm 3.84,95 \%$ C.I.: 69.95 to $85.64 \%)$ and best

740 performance when saccades were made after the working memory task $(93.28 \% \pm 1.844 \mathrm{SE}, 95 \%$

741 C.I.: 89.45 to 97.12 ). Panel b shows the relationship of the sensitivity measured d-prime with

742 direction of laterality, while panels $\mathrm{c}$ and d show relationships between percent correct accuracy

743 and d-prime respectively with degree of laterality as expressed by the absolute value of laterality

744 quotients.

745

746 Figure 4. Scene Recognition Memory Performance as a Function of Laterality and Eye Task

747 Timing. There was a significant relationship in recognition percent correct performance as a

748 function of direction of laterality during the condition of fixation before the recognition memory

749 task (non-zero slope $=0.08738 \pm 0.03277 \mathrm{SE}, \mathrm{F}_{(1,21)}=7.111, \mathrm{p}=0.0144$, grey line and grey boxes,

750 panel a). Overall mean performance among the conditions differed $\left(\mathrm{F}_{(3,106)}=4.721, \mathrm{p}=0.0039\right)$ with

751 best recognition memory performance during the condition of fixation before the working memory

752 task $(70.08 \% \pm 3.899$ SE, $95 \%$ C.I.: 62.12 to 78.03 , blue line and blue circles, panel a) and lowest

753 recognition memory performance during the condition when saccades were made before the

754 working memory task $(60.67 \% \pm 4.06,95 \%$ C.I.: 52.38 to 68.96 , red line and red triangles, panel

755 a). Panel $b$ shows the relationship of the sensitivity measured d-prime with direction of laterality,

756 while panels $\mathrm{c}$ and $\mathrm{d}$ show relationships between percent correct accuracy and d-prime respectively

757 with degree of laterality as expressed by the absolute value of laterality quotients. 
761

762

763

764

765

766

767

768

769

770

771

772

773

774

775

776

777

778

779

780

781

782

783

784

785

786

787

788

789

790

791

792

793

794

\section{References}

Baddeley A. 2003. Working memory: looking back and looking forward. Nature reviews neuroscience 4:829.

Brewer JB, Zhao Z, Desmond JE, Glover GH, and Gabrieli JD. 1998. Making memories: brain activity that predicts how well visual experience will be remembered. Science 281:11851187.

Brewin CR. 2014. Episodic memory, perceptual memory, and their interaction: foundations for a theory of posttraumatic stress disorder. Psychol Bull 140:69-97. 10.1037/a0033722

Brown AL, and Scott MS. 1971. Recognition memory for pictures in preschool children. Journal of Experimental Child Psychology 11:401-412.

Brunye TT, Mahoney CR, Augustyn JS, and Taylor HA. 2009. Horizontal saccadic eye movements enhance the retrieval of landmark shape and location information. Brain Cogn 70:279-288. 10.1016/j.bandc.2009.03.003

Burgess N, Maguire EA, and O'Keefe J. 2002. The human hippocampus and spatial and episodic memory. Neuron 35:625-641.

Christman S, and Butler M. 2005. Bilateral eye movements impair the encoding and enhance the retrieval of episodic memories. 46th annual meeting of the Psychonomic Society, Toronto, Ontario.

Christman SD, Garvey KJ, Propper RE, and Phaneuf KA. 2003. Bilateral eye movements enhance the retrieval of episodic memories. Neuropsychology 17:221-229.

Christman SD, and Propper RE. 2001. Superior episodic memory is associated with interhemispheric processing. Neuropsychology 15:607.

Christman SD, Propper RE, and Dion A. 2004. Increased interhemispheric interaction is associated with decreased false memories in a verbal converging semantic associates paradigm. Brain Cogn 56:313-319. 10.1016/j.bandc.2004.08.005

Chu O, Abeare CA, and Bondy MA. 2012. Inconsistent vs consistent right-handers' performance on an episodic memory task: evidence from the California Verbal Learning Test. Laterality 17:306-317. 10.1080/1357650X.2011.568490

Cowan N. 1999. An embedded-processes model of working memory. Models of working memory: Mechanisms of active maintenance and executive control 20:506.

Dragovic M. 2004. Towards an improved measure of the Edinburgh Handedness Inventory: A one-factor congeneric measurement model using confirmatory factor analysis. Laterality: Asymmetries of Body, Brain and Cognition 9:411-419. 
795

796

797

798

799

800

801

802

803

804

805

806

807

808

809

810

811

812

813

814

815

816

817

818

819

820

821

822

823

824

825

826

827

828
Edlin JM, Leppanen ML, Fain RJ, Hackländer RP, Hanaver-Torrez SD, and Lyle KB. 2015. On the use (and misuse?) of the Edinburgh Handedness Inventory. Brain and cognition 94:44-51.

Edlin JM, and Lyle KB. 2013. The effect of repetitive saccade execution on the attention network test: enhancing executive function with a flick of the eyes. Brain $\operatorname{Cogn}$ 81:345351. 10.1016/j.bandc.2012.12.006

Ellmore TM, Feng A, Ng K, Dewan L, and Root JC. 2015. The Effects of Changing Attention and Context in an Awake Offline Processing Period on Visual Long-Term Memory. Front Psychol 6:1902. 10.3389/fpsyg.2015.01902

Ellmore TM, Ng K, and Reichert CP. 2017. Early and late components of EEG delay activity correlate differently with scene working memory performance. PLoS One 12:e186072. 10.1371/journal.pone.0186072

Eriksen A, Lorås H, Vorland Pedersen A, and Sigmundsson H. 2018. Proximal-Distal Motor Control in Skilled Drummers: The Effect on Tapping Frequency of Mechanically Constraining Joints of the Arms in Skilled Drummers and Unskilled Controls.

Fan J, Gu X, Guise KG, Liu X, Fossella J, Wang H, and Posner MI. 2009. Testing the behavioral interaction and integration of attentional networks. Brain Cogn 70:209-220. 10.1016/j.bandc.2009.02.002

Golby AJ, Poldrack RA, Brewer JB, Spencer D, Desmond JE, Aron AP, and Gabrieli JD. 2001. Material-specific lateralization in the medial temporal lobe and prefrontal cortex during memory encoding. Brain 124:1841-1854.

Holcombe A, Nguyen E, and Goodbourn P. 2017. Implied Reading Direction and Prioritization of Letter Encoding.

Kielar A, Deschamps T, Chu R, Jokel R, Khatamian Y, Chen JJ, and Meltzer J. 2016. Identifying Dysfunctional Cortex: Dissociable Effects of Stroke and Aging on Resting State Dynamics in MEG and fMRI.

Lyle KB, and Edlin JM. 2015. Why does saccade execution increase episodic memory retrieval? A test of the top-down attentional control hypothesis. Memory 23:187-202. $10.1080 / 09658211.2013 .877487$

Lyle KB, Hanaver-Torrez SD, Hacklander RP, and Edlin JM. 2012. Consistency of handedness, regardless of direction, predicts baseline memory accuracy and potential for memory enhancement. J Exp Psychol Learn Mem Cogn 38:187-193. 10.1037/a0024831

Lyle KB, and Jacobs NE. 2010. Is saccade-induced retrieval enhancement a potential means of improving eyewitness evidence? Memory 18:581-594. 10.1080/09658211.2010.493891 
829 Lyle KB, Logan JM, and Roediger HL, 3rd. 2008a. Eye movements enhance memory for 830 individuals who are strongly right-handed and harm it for individuals who are not. $831 \quad$ Psychon Bull Rev 15:515-520.

832 Lyle KB, and Martin JM. 2010. Bilateral saccades increase intrahemispheric processing but not

833

834

835

836

837

838

839

840

841

842

843

844

845

846

847

848

849

850

851

852

853

854

855

856

857

858

859

860

861

862

863

Nieuwenhuis S, Elzinga BM, Ras PH, Berends F, Duijs P, Samara Z, and Slagter HA. 2013. Bilateral saccadic eye movements and tactile stimulation, but not auditory stimulation, enhance memory retrieval. Brain Cogn 81:52-56. 10.1016/j.bandc.2012.10.003

Nyberg L, Cabeza R, and Tulving E. 1996. PET studies of encoding and retrieval: The HERA model. Psychon Bull Rev 3:135-148. 10.3758/BF03212412

Oldfield RC. 1971. The assessment and analysis of handedness: the Edinburgh inventory. Neuropsychologia 9:97-113.

Parker A, and Dagnall N. 2007. Effects of bilateral eye movements on gist based false recognition in the DRM paradigm. Brain Cogn 63:221-225. 10.1016/j.bandc.2006.08.005

Parker A, and Dagnall N. 2010. Effects of handedness and saccadic bilateral eye movements on components of autobiographical recollection. Brain $\operatorname{Cogn}$ 73:93-101. 10.1016/j.bandc.2010.03.005

Parker A, Parkin A, and Dagnall N. 2017. Effects of handedness \& saccadic bilateral eye movements on the specificity of past autobiographical memory \& episodic future thinking. Brain Cogn 114:40-51. 10.1016/j.bandc.2017.03.006

Parker A, Relph S, and Dagnall N. 2008. Effects of bilateral eye movements on the retrieval of item, associative, and contextual information. Neuropsychology 22:136-145. 10.1037/0894-4105.22.1.136

Peyrin C, Baciu M, Segebarth C, and Marendaz C. 2004. Cerebral regions and hemispheric specialization for processing spatial frequencies during natural scene recognition. An event-related fMRI study. Neuroimage 23:698-707. 10.1016/j.neuroimage.2004.06.020 
864 Prichard EC, and Christman SD. 2017. Inconsistent-handed advantage in episodic memory

865

866

867

868

869

870

871

872

873

874

875

876

877

878

879

880

881

882

883

884

885

886

887

888

889

890

891

892

893

894

895 896 extends to paragraph-level materials. Memory 25:1063-1071. $10.1080 / 09658211.2016 .1257725$

Propper RE, and Christman SD. 2008. Interhemispheric Interaction and Saccadic Horizontal Eye MovementsImplications for Episodic Memory, EMDR, and PTSD. Journal of EMDR Practice and Research 2:269-281.

Propper RE, Christman SD, and Phaneuf KA. 2005. A mixed-handed advantage in episodic memory: a possible role of interhemispheric interaction. Mem Cognit 33:751-757.

Propper RE, Patel N, Christman SD, and Carlei C. 2017. Superior episodic memory in inconsistent-handers: a replication and extension using fNIRS. Memory 25:1390-1395. 10.1080/09658211.2017.1309051

Propper RE, Pierce J, Geisler MW, Christman SD, and Bellorado N. 2007. Effect of bilateral eye movements on frontal interhemispheric gamma EEG coherence: implications for EMDR therapy. J Nerv Ment Dis 195:785-788. 10.1097/NMD.0b013e318142cf73

Rice M, and M. Gosser S. 2015. Efficiency of unimanual and bimanual reach in persons with and without stroke.

Samara Z, Elzinga BM, Slagter HA, and Nieuwenhuis S. 2011. Do Horizontal Saccadic Eye Movements Increase Interhemispheric Coherence? Investigation of a Hypothesized Neural Mechanism Underlying EMDR. Front Psychiatry 2:4. 10.3389/fpsyt.2011.00004

Shepard RN. 1967. Recognition memory for words, sentences, and pictures. Journal of verbal Learning and verbal Behavior 6:156-163.

Standing L. 1973. Learning 10000 pictures. The Quarterly journal of experimental psychology 25:207-222.

Standing L, Conezio J, and Haber RN. 1970. Perception and memory for pictures: Single-trial learning of 2500 visual stimuli. Psychonomic Science 19:73-74.

Stanislaw H, and Todorov N. 1999. Calculation of signal detection theory measures. Behav Res Methods Instrum Comput 31:137-149.

Sternberg S. 1966. High-speed scanning in human memory. Science 153:652-654.

Stevens WD, Kahn I, Wig GS, and Schacter DL. 2012. Hemispheric asymmetry of visual scene processing in the human brain: evidence from repetition priming and intrinsic activity. Cereb Cortex 22:1935-1949. 10.1093/cercor/bhr273

Stickgold R. 2002. EMDR: a putative neurobiological mechanism of action. J Clin Psychol 58:61-75. 
897 Williams S. 2010. A major revision of the Edinburgh Handedness Inventory. Colchester, Essex, $898 \quad$ United Kingdom, https://doi.org/10.13140/RG.2.2.33298.25284.

899 Williams SM. 1986. Factor analysis of the Edinburgh Handedness Inventory. Cortex: A Journal $900 \quad$ Devoted to the Study of the Nervous System and Behavior 22:325-326.

901 Xiao J, Hays J, Ehinger K, Oliva A, and Torralba A. 2010. SUN database: Large-scale scene 902 recognition from abbey to zoo. 2010 IEEE Conference on Computer Vision and Pattern 904 Recognition (CVPR). San Francisco, CA: IEEE. p 3485-3492. 


\section{Figure 1}

\section{Example Scene Working and Recognition Memory Trials}

Each working memory trial (a) consisted of an encoding phase of five scene stimuli, each presented for $2 \mathrm{sec}$, followed by a $6 \mathrm{sec}$ delay period of crosshair fixation and a $2 \mathrm{sec}$ presentation of either a positive probe (scene from the encoding set) or a negative probe (new scene). On each trial, there was a 50\% chance of a positive probe appearing. Each recognition memory trial (b) consisted of alternating presentation of a scene stimuli for $2 \mathrm{sec}$ followed by a black screen for $2 \mathrm{sec}$. Old and new scenes were randomly intermixed. Subjects were instructed to press a green button if they had seen a stimulus in any of the previous working memory trials and a red button if they had never seen the stimulus before. In this example, image numbers from the set of scene stimuli are listed rather than the actual scene images to illustrate how scenes during encoding could be presented as a positive probe (a) or as old stimuli in the recognition task (b). 

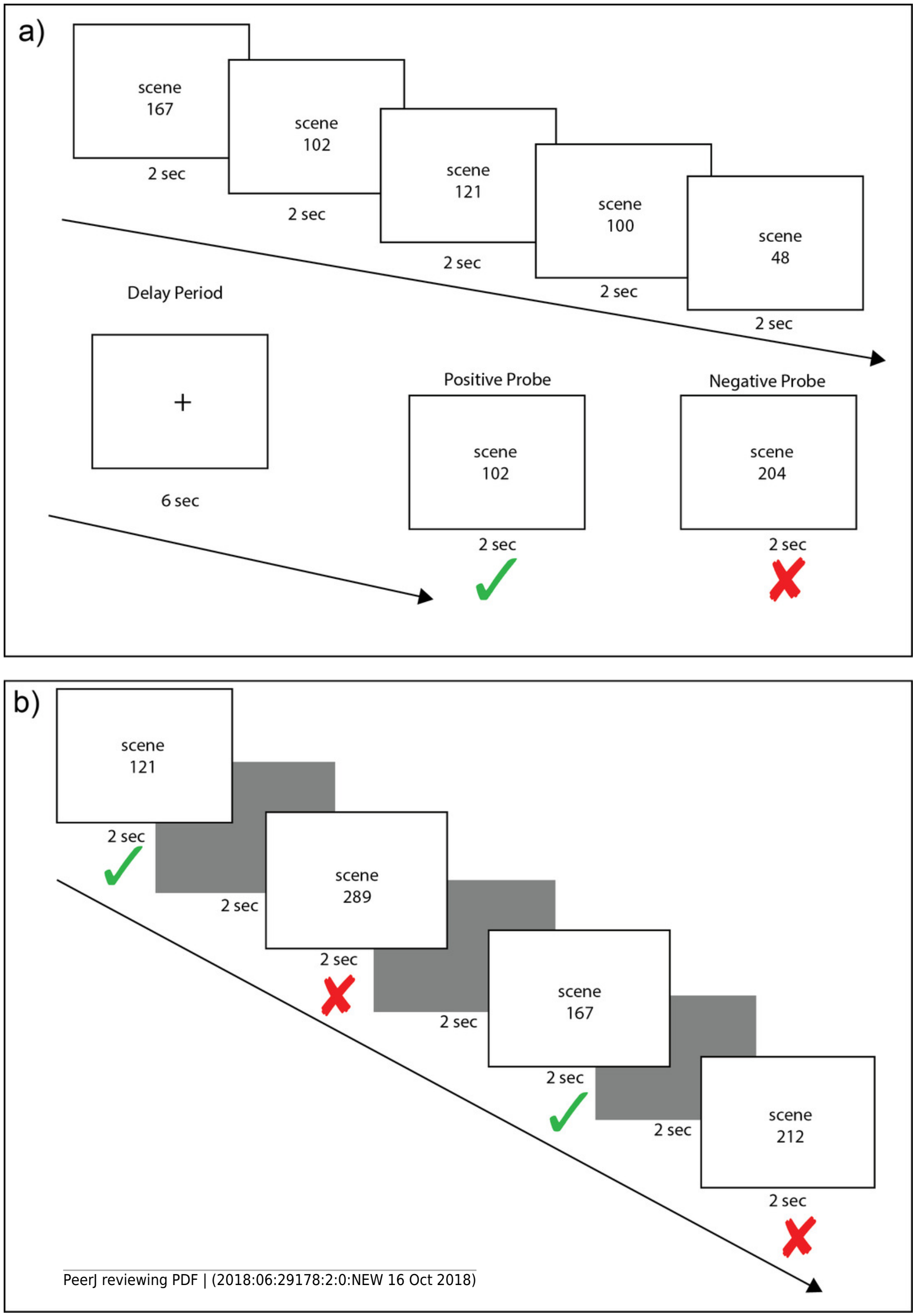


\section{Figure 2}

The Saccade and Fixation Eye Tasks

The saccade task (top left) required subjects to alternate for 30 seconds looking toward the left and right as a white disc moved back and forth across the screen. Example eye traces (top right) show saccadic movements with large periodic deviations in the $\mathrm{x}$ position (horizontal) trace with a stable y position (vertical) trace. The fixation task (bottom left) required subjects to maintain fixation on a center disc as it changed color. Example eyetracking traces (bottom right) show fixation, with minimal deviations in the $\mathrm{x}$ (horizontal) and y position (vertical) traces. 


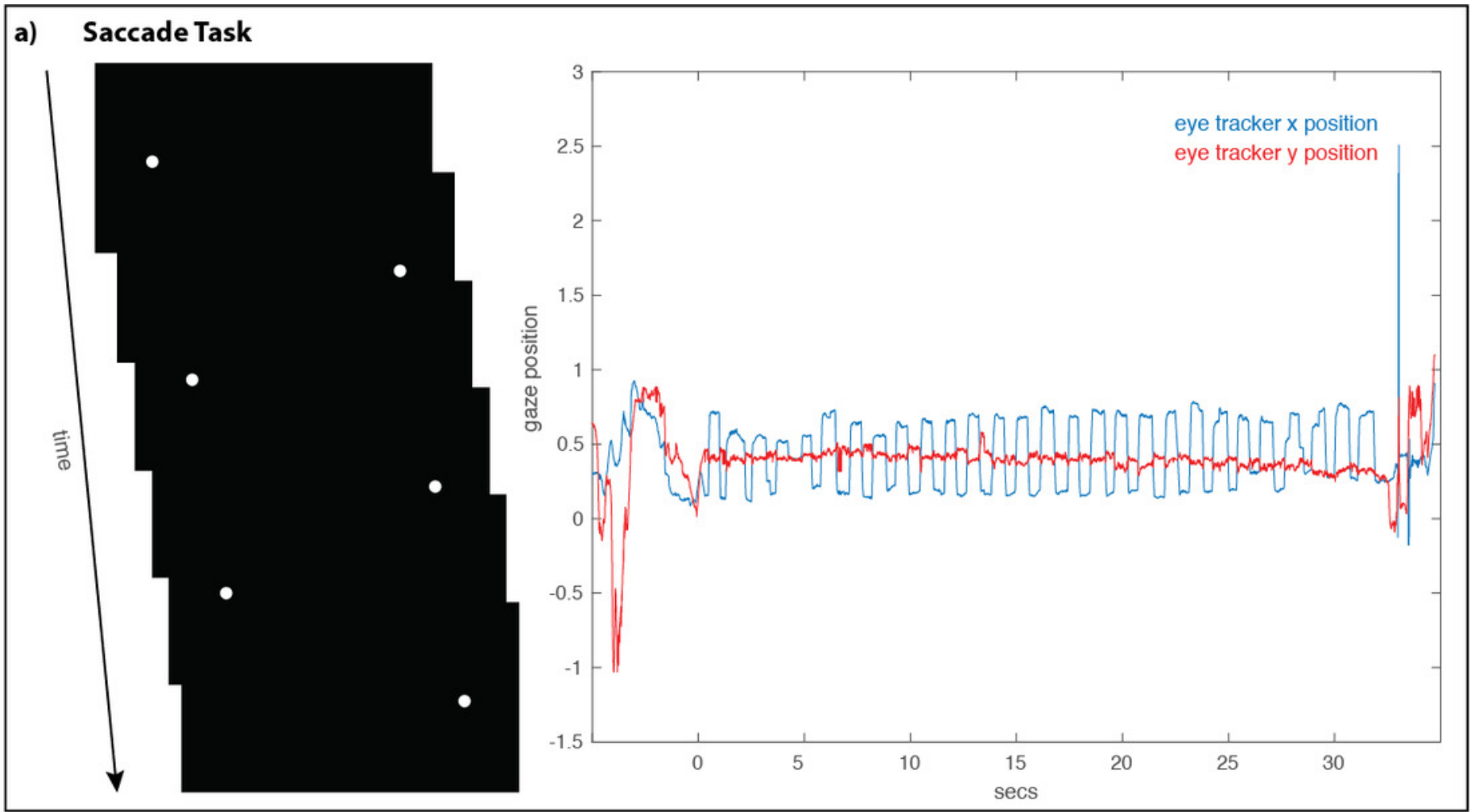

\section{b) Fixation Task}

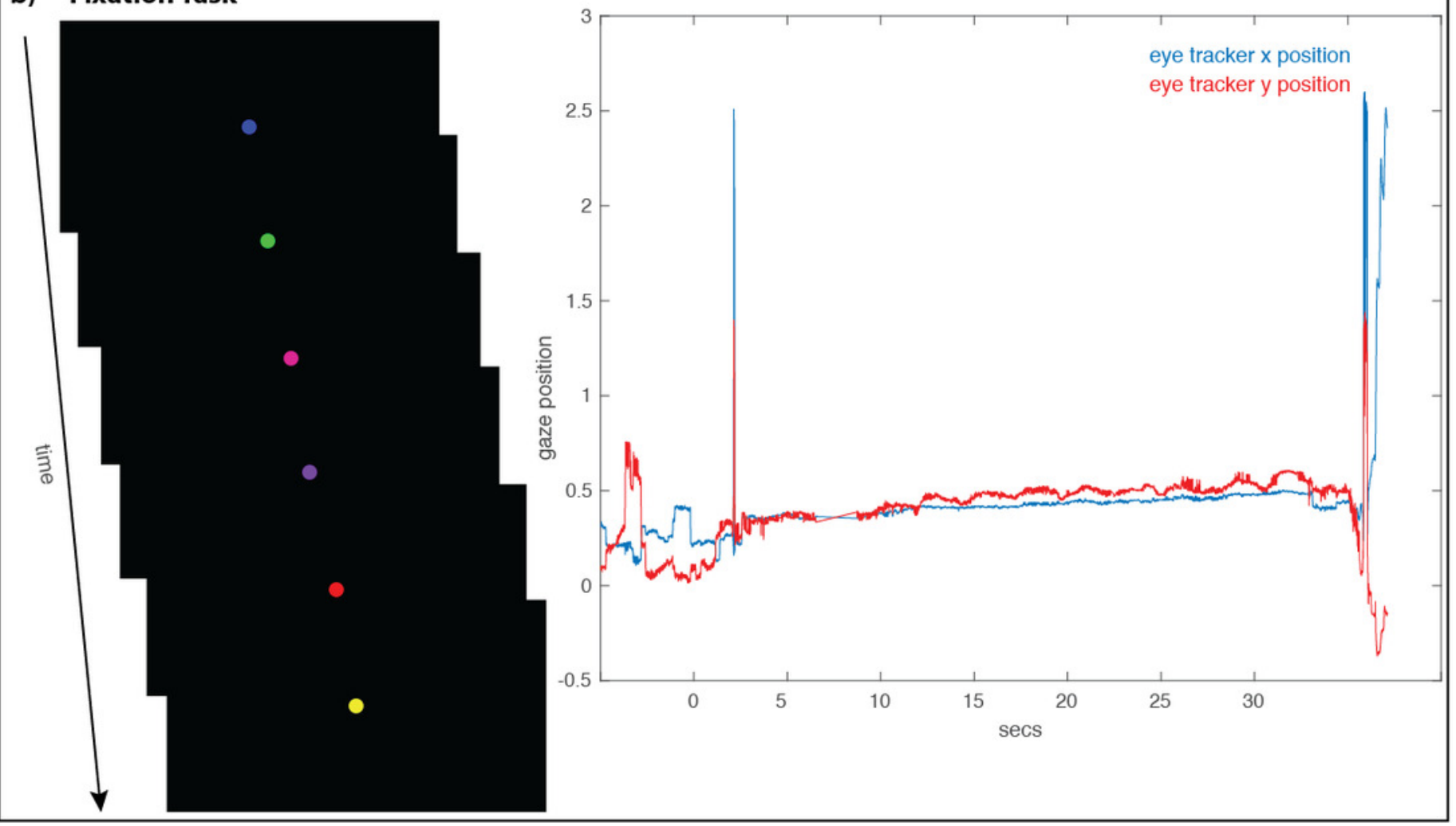




\section{Figure $\mathbf{3}$ (on next page)}

Scene Working Memory Performance as a Function of Laterality and Eye Task Timing

There was a significant relationship in working memory percent correct performance as a function of direction of laterality when saccades were made before the working memory task (non-zero slope of $0.1029 \pm 0.04365 \mathrm{SE}, \mathrm{F}_{(1,30)}=5.56, p=0.0251$, red line and red triangles, panel a). Overall mean performance among conditions differed (non-zero intercepts, $\left.F_{(3,106)}=6.164, p=0.0007\right)$ with lowest working memory percent correct performance obtained when saccades were made before the working memory task $(77.79 \% \pm 3.84,95 \%$ C.I.: 69.95 to $85.64 \%$ ) and best performance when saccades were made after the working memory task (93.28\% $\pm 1.844 \mathrm{SE}, 95 \%$ C.I.: 89.45 to 97.12 ). Panel b shows the relationship of the sensitivity measured $d$-prime with direction of laterality, while panels $c$ and $d$ show relationships between percent correct accuracy and d-prime respectively with degree of laterality as expressed by the absolute value of laterality quotients 
PeerJ
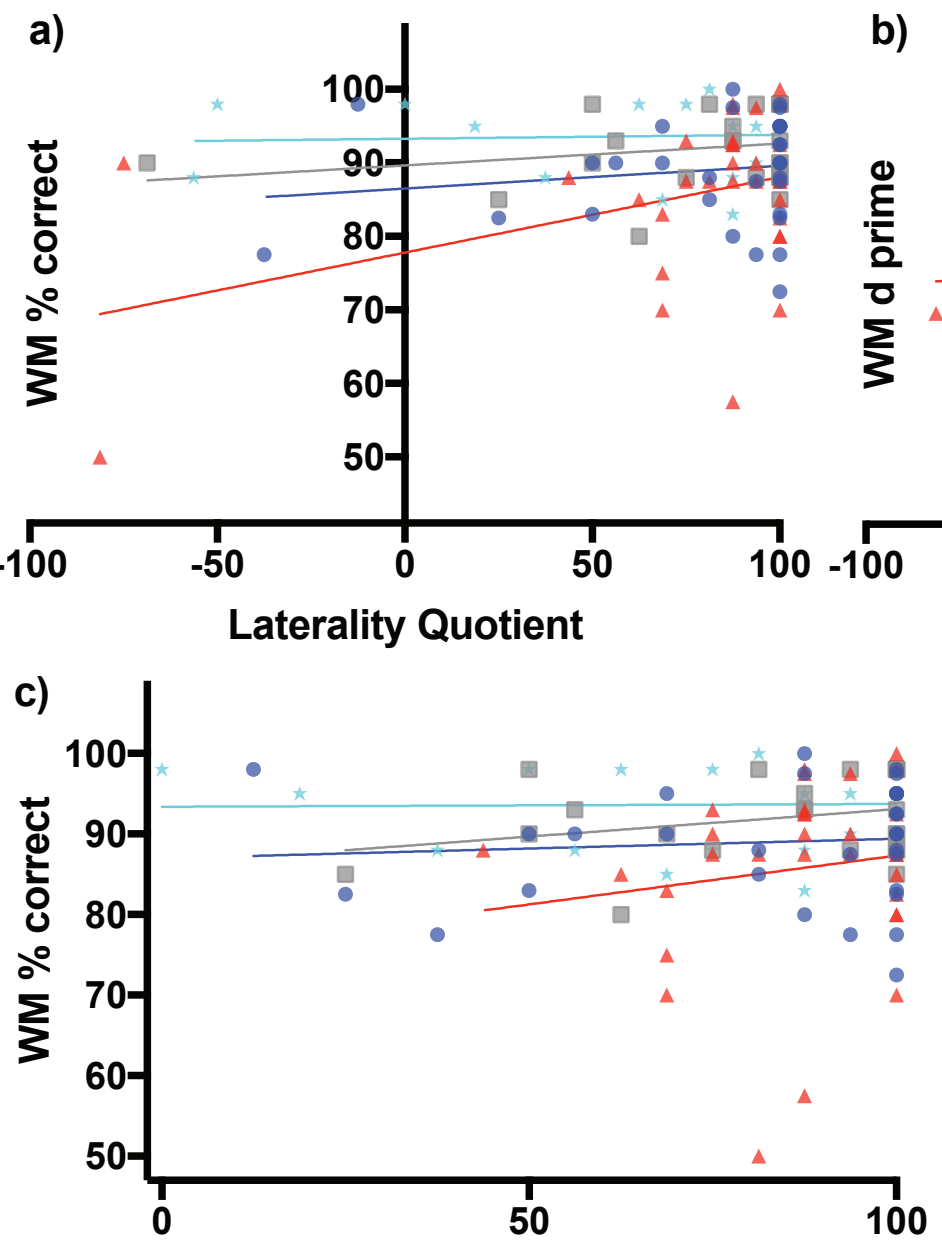

Absolute Value of Laterality Quotient
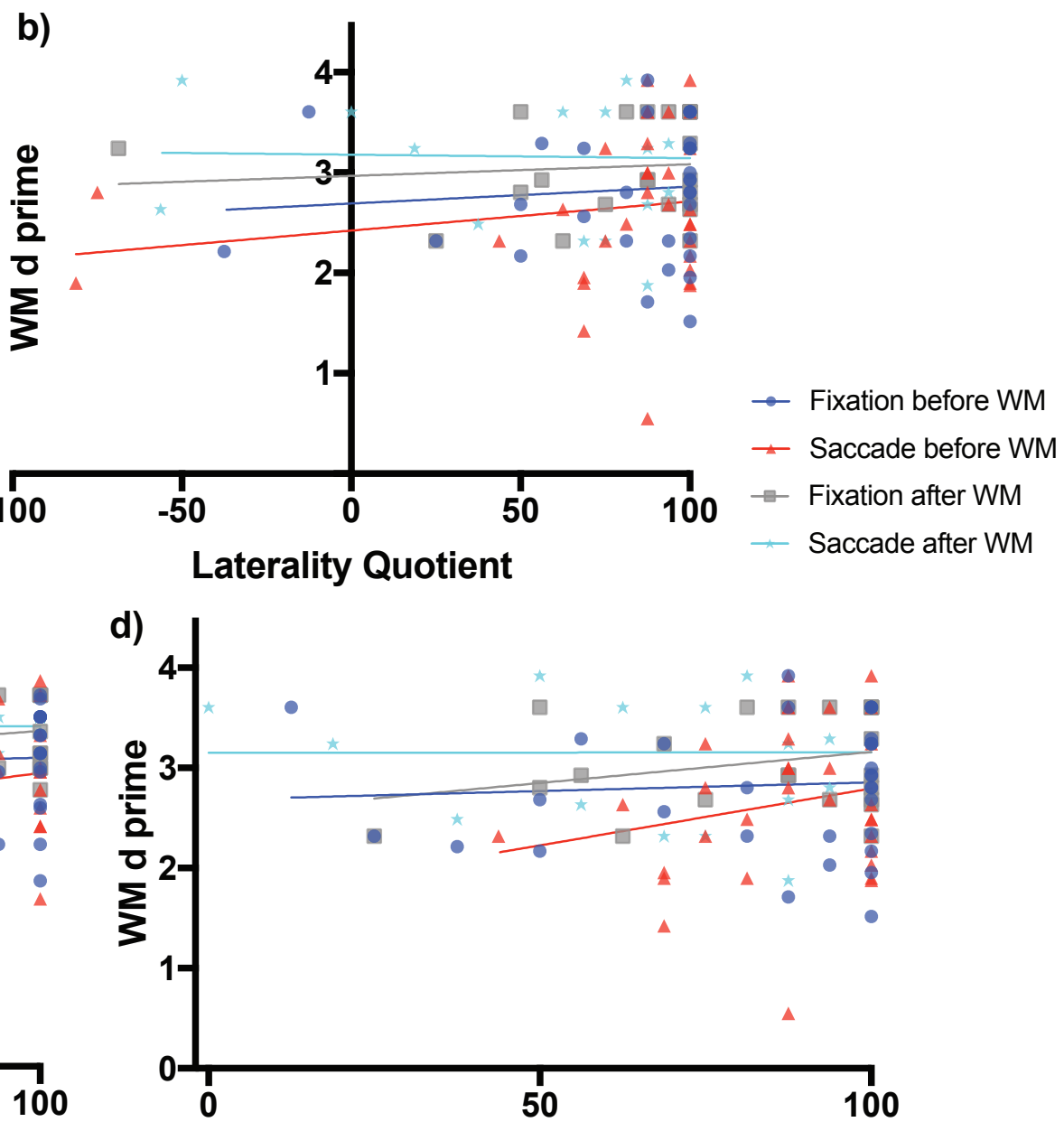

Absolute Value of Laterality Quotient 


\section{Figure 4 (on next page)}

Scene Recognition Memory Performance as a Function of Laterality and Eye Task Timing

There was a significant relationship in recognition percent correct performance as a function of direction of laterality during the condition of fixation before the recognition memory task (non-zero slope $=0.08738 \pm 0.03277 \mathrm{SE}, \mathrm{F}_{(1,21)}=7.111, p=0.0144$, grey line and grey boxes, panel a). Overall mean performance among the conditions differed $\left(F_{(3,106)}=4.721, p=0.0039\right)$ with best recognition memory performance during the condition of fixation before the working memory task $(70.08 \% \pm 3.899 \mathrm{SE}, 95 \%$ C.I.: 62.12 to 78.03 , blue line and blue circles, panel a) and lowest recognition memory performance during the condition when saccades were made before the working memory task $(60.67 \% \pm 4.06,95 \%$ C.I.: 52.38 to 68.96, red line and red triangles, panel a). Panel b shows the relationship of the sensitivity measured d-prime with direction of laterality, while panels $\mathrm{c}$ and $\mathrm{d}$ show relationships between percent correct accuracy and d-prime respectively with degree of laterality as expressed by the absolute value of laterality quotients. 


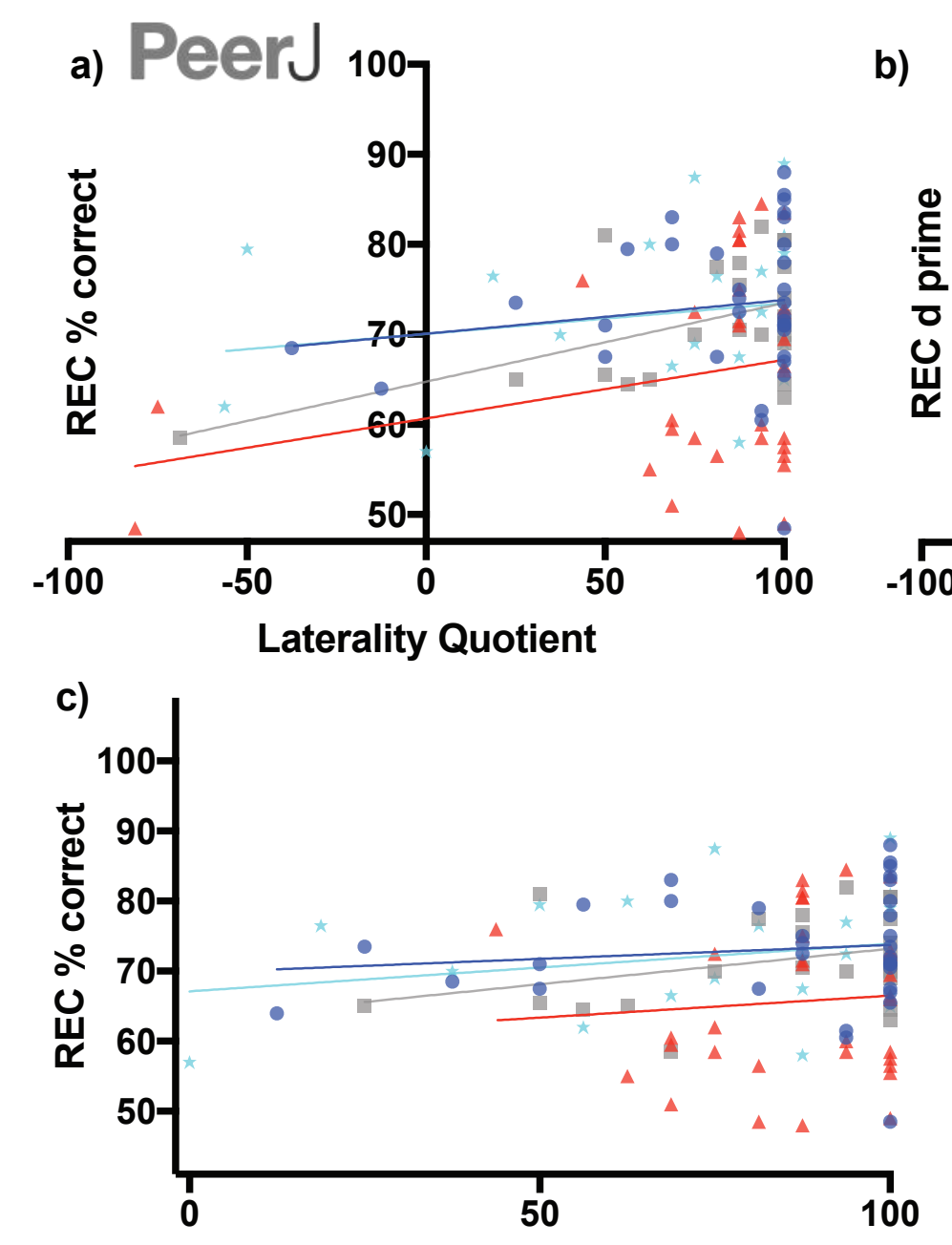

Absolute Value of Laterality Quotient b) Manuscript to be reviewed

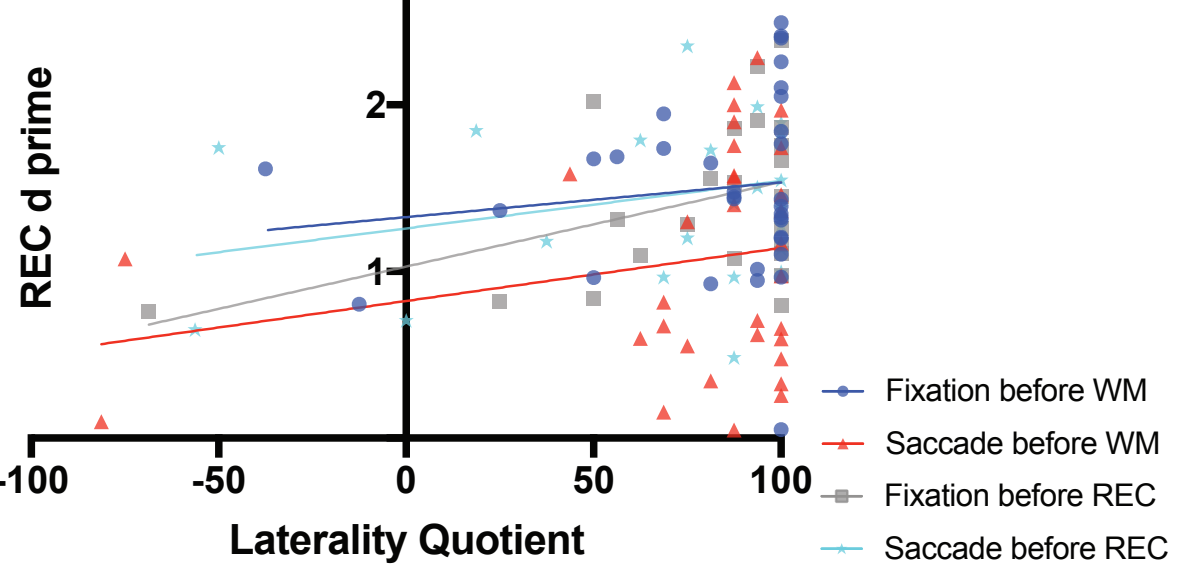

d)

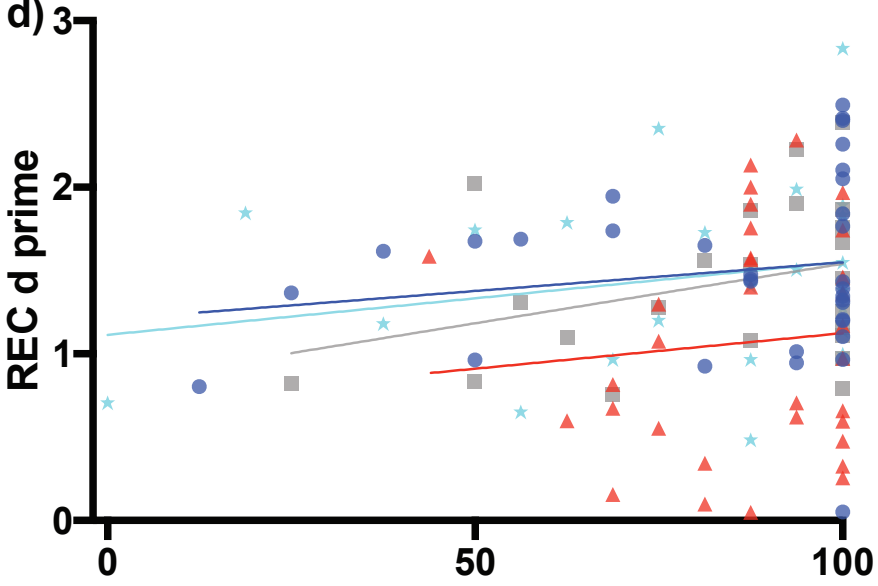

Absolute Value of Laterality Quotient 